\title{
Development of fludarabine formulations in the treatment of chronic lymphocytic leukemia
}

This article was published in the following Dove Press journal:

Drug Design, Development and Therapy

13 October 2009

Number of times this article has been viewed

\begin{abstract}
Ann Janssens
Marc Boogaerts

Gregor Verhoef

Department of Hematology, University Hospitals Leuven, Campus Gasthuisberg, Leuven, Belgium
\end{abstract}

Correspondence: Ann Janssens Department of Hematology, University Hospitals Leuven, Campus Gasthuisberg, Leuven, Belgium

Tel +32 I6 346880

Fax +32 I6 346881

Email ann.janssens@uz.kuleuven.ac.be

\begin{abstract}
Fludarabine is an antineoplastic agent used in the treatment of hematological malignancies, particularly chronic lymphocytic leukemia (CLL) and indolent B-cell lymphoma. Because of its immunosuppressive effects, fludarabine has been added to reduced intensity conditioning regimens. The oral formulation of fludarabine has become widely available. Pharmacokinetic studies have shown that an oral dose of $40 \mathrm{mg} / \mathrm{m}^{2} / \mathrm{d}$ would provide systemic drug exposure similar to the standard intravenous (IV) dose of $25 \mathrm{mg} / \mathrm{m}^{2} / \mathrm{d}$. The oral dose can be taken once daily without any dietary restrictions. Dose adjustments are mandatory in patients with renal impairment to avoid increased toxicity. Several noncomparative trials in previously untreated and treated patients with CLL have shown that treatment with the oral formulation demonstrates similar efficacy compared to historical control groups treated with the IV formulation. The tolerability profile of oral fludarabine seems similar to that of the IV formulation. Myelosuppression and infectious complications are the most frequently reported adverse events. Gastrointestinal toxicity is more frequent with the oral formulation, but is usually of mild or moderate severity. Although oral fludarabine makes treatment more convenient, health care workers must be aware of the compliance behavior of each patient.
\end{abstract}

Keywords: CLL, treatment, oral fludarabine, intravenous fludarabine

\section{Introduction}

Nucleoside analogues (NA) constitute an important class of antimetabolites used in the treatment of hematological malignancies and more recently in solid tumors. The NA family includes various pyrimidine and purine analogues (PA). Cytosine arabinoside and gemcitabine are well known pyrimidine analogues. The two oldest PA are 6-mercaptopurine and 6-thioguanine, available in an oral formulation and used especially in the treatment of acute leukemias. The next generation of PAs consist of cladribine, pentostatin, and fludarabine. These molecules have been available worldwide since the 1990s. Three novel PA, clofarabine, nelarabine, and forodesine, have been introduced in clinical trials and are entering daily practice.

For several decades the standard treatment for chronic lymphocytic leukemia (CLL) has been single-agent chlorambucil or alkylator-based combination treatments. These regimens were very effective in controlling symptoms and tumor burden although complete response (CR) rate was low. ${ }^{1}$

Fludarabine is the PA most extensively studied in the treatment of indolent B-cell malignancies. Fludarabine monotherapy has yielded high response rates in untreated and treated CLL patients as well. ${ }^{2-4}$ Later attempts to further improve CR and response duration have been explored with the use of fludarabine in combination 
with chemotherapeutic agents and/or nonchemotherapeutic agents like monoclonal antibodies.

An oral formulation of fludarabine has been developed. In 2001, the UK's National Institute for Clinical Excellence (NICE) recommended the use of the oral formulation instead of the intravenous (IV) formulation. Since then oral fludarabine became available in many areas of Europe and Canada. In December 2008, the US Food and Drug Administration (FDA) approved oral fludarabine for the treatment of relapsed CLL.

\section{Pharmacodynamic properties}

Fludarabine or 5'-monophosphate of F-ara-A functions as a prodrug. After the dephosphorylation of fludarabine to F-ara-A by the 5'-nucleotidase activity on erythrocytes, endothelium, and vascular linings of large body organs, F-ara-A is taken into cells by nucleoside-specific membrane transporters. After entering the cell F-ara-A is rephosphorylated to monophosphate by deoxycytidine kinase and subsequently to diphosphate and triphosphate. The triphosphate F-ara-ATP appears to be the only metabolite with cytotoxic activity. The principal mechanism of action of F-ara-ATP is inhibition of DNA and RNA synthesis. F-ara-ATP inhibits DNA polymerases, DNA primase, DNA ligase, and also ribonucleotide reductase. Inhibition of ribonucleotide reductase lowers the cellular concentration of the normal pool of deoxynucleotides, changing the ratio of F-ara-ATP to deoxyadenosine 5'-triphosphate (dATP) and promoting the incorporation of F-ara-AMP in DNA. Inhibition of the other mentioned enzymes makes further DNA extension and ligation to other DNA fragments impossible. F-ara-AMP seems also resistant to removal by proofreading activities. This makes F-ara-AMP an effective chain terminator leading to inactivation of DNA synthesis and accumulation of DNA breaks followed by initiation of programmed cell death or apoptosis (p53-dependent and -independent). Quiescent lymphocytes are continually breaking and rejoining their DNA, which explains why PA can express cytotoxicity in malignancies where proliferation is not impressive. F-ara-ATP seems the sole PA capable of inhibiting RNA synthesis. ${ }^{5}$ Incorporation of F-ara-AMP in RNA results in repression of gene transcription leading to reduced expression of proteins that may be important for cell survival. The lack of survival proteins can induce cell death in dividing as well as resting cells. ${ }^{6}$

\section{Pharmacokinetic properties}

Two bioavailability studies of oral fludarabine have been reported. The first study tested a liquid formulation consisting of the injectable solution and demonstrated a $75 \%$ bioavailability. $^{7}$ The second study tested tablets with immediate-release fludarabine and suggested $55 \%$ oral availability that was dose independent. The intra-individual variation in bioavailability was low although bioavailability ranged from $30 \%$ to $80 \%$ between patients. F-ara-A could be detected in plasma 15 to 30 minutes after oral dosing with reaching a maximum plasma concentration $\left(\mathrm{C}_{\max }\right)$ approximately after 1.1-1.2 hours. With the $50 \mathrm{mg}$ IV dose, $\mathrm{C}_{\max }$ is reached immediately at the end of the infusion and is three times higher than $\mathrm{C}_{\text {max }}$ with the $90 \mathrm{mg}$ oral dose. However, oral dose $\mathrm{C}_{\max }$ was similar to the IV dose $\mathrm{C}_{\max }$ when measured 30 to 60 minutes after termination of the infusion. The linear dose proportional increase in area under the plasma concentration-time curve (AUC (0-24 hours)) seen with different IV dosages was also noted for the oral administration. The AUC (0-24 hours) attained after a $90 \mathrm{mg}$ oral dose was similar to that attained after a $50 \mathrm{mg}$ IV dose. ${ }^{8}$ It has also been shown that pharmacokinetics do not significantly differ when oral fludarabine is taken with or without food. The time to $\mathrm{C}_{\max }$ was slightly extended by the presence of food but the terminal half-life was unaffected. ${ }^{9}$

Elimination of F-ara-A is largely by renal excretion. Approximately $37 \% \pm 5 \%$ of a 30 -minute infusion was recovered in the urine within 24 hours, rising to $57 \% \pm 7 \%$ after 72 hours. Similar F-ara-A elimination kinetics were observed after oral administration. There is heterogeneity among individuals with respect to the rate of F-ara-ATP accumulation and retention leading to a half-life ranging from a few hours to a few days. F-ara-ATP is a relatively long-lived active metabolite, which makes daily administration convenient. ${ }^{5}$ A pharmacokinetic study of oral fludarabine in relapsed indolent B-cell non-Hodgkin lymphoma showed that time to $\mathrm{C}_{\max }$, $\mathrm{AUC}$ (0-24 hours), elimination time, and bioavailabily in Japanese patients were comparable with the data obtained in Caucasian patients suggesting no ethnic differences. ${ }^{10}$

All these studies have suggested that a once-daily oral dose of $40 \mathrm{mg} / \mathrm{m}^{2} / \mathrm{d}$ given as immediate-release tablets will provide a similar systemic exposure as an IV dose of $25 \mathrm{mg} / \mathrm{m}^{2} / \mathrm{d}$, independently of food intake. ${ }^{11}$

\section{Therapeutic efficacy}

No randomized controlled trials (RCT) have compared the efficacy of oral versus IV fludarabine, neither in CLL nor in indolent lymphoma. Some observational studies with oral fludarabine reporting safety and efficacy were found. Some data were retrieved from published full papers, some from abstracts. Efficacy and safety data were compared with 
historical data concerning treatment with IV fludarabine (Table 1).

\section{Single-agent fludarabine in previously treated CLL}

One of the earliest published single-center noncomparative studies reported on the outcome of treatment with singleagent fludarabine IV in 68 patients with previously treated CLL. CR was achieved in $13 \%$ and partial response (PR) in $44 \%$ of patients. The authors concluded that fludarabine was a new agent with marked activity against CLL and excellent tolerance. The response rates were superior to those of other single-agents and comparable with the results of combination regimens in previously treated patients. ${ }^{2}$

In a prospective multicenter phase II clinical trial, relapsed/refractory CLL patients were treated with oral fludarabine $40 \mathrm{mg} / \mathrm{m}^{2}$ for five days every four weeks for a total of six to eight cycles. Seventy-eight patients were evaluable. Median age was 63.4 years (range 55-72 years). Overall response (OR) rate and $\mathrm{CR}$ according to National Cancer Institute-sponsored Working Group (NCI-WG) criteria were $51.3 \%$ and $17.9 \%{ }^{12}$ Grade 3-4 neutropenia was seen in $53.8 \%$ of patients although grade 3-4 infections were reported in only $7.7 \%$ of cases. Gastrointestinal toxicity was more common than previously reported with the IV formulation but was generally mild to moderate. Grade 1-2 nausea/vomiting was seen in $37 \%$ of patients with only $1.3 \%$ complaining of grade 3 nausea/vomiting. Grade 1-2 diarrhea was noticed in $34.6 \%$ of patients with grade 3 observed in $3.8 \%$ of patients. ${ }^{13}$

\section{Single-agent fludarabine in untreated CLL} A meta-analysis selected five CLL RCTs comparing singleagent PA with alkylator-based regimens as front-line treatment. OR, CR, and progression-free survival (PFS) were in favor for the PA although overall survival (OS) was identical. Although the incidence of grade 3-4 neutropenia was significantly higher in patients treated with PA infections were not significantly increased. ${ }^{4}$ One of these trials compared fludarabine IV with chlorambucil. Efficacy and safety data for the fludarabine-treated patients were as follows: OR $63 \%$ and CR 20\% (NCI-WG criteria), median PFS, and OS 20 and 66 months, respectively. Grade 3-4 neutropenia was seen in $27 \%$ of patients, with grade 3-4 infections diagnosed in $16 \%$ of patients. $^{14}$

Eighty-one previously untreated CLL patients with a median age of 61.2 years (range 30-75 years) were treated with oral fludarabine $40 \mathrm{mg} / \mathrm{m}^{2} / \mathrm{d}$ for five days every four weeks for a total of six or eight cycles. OR was $80.2 \%$ with a $\mathrm{CR}$ of $12.3 \%$ (NCI-WG criteria). Median time to progression was 841 days. Grade 3-4 neutropenia and infections were reported in $32.1 \%$ and $4.9 \%$ of patients, respectively. Gastrointestinal toxicity was more common with the oral formulation, but was generally mild and did not require treatment. Forty-two percent of patients experienced any degree of diarrhea with grade $3-4$ in $6.2 \%$ of patients. Any degree of nausea or vomiting was experienced by $38.2 \%$ of patients, with only $1.2 \%$ grade $3-4$. This toxicity did not cause compliance problems or withdrawal from the study. Oral fludarabine did not adversely affect quality of life and may even improve emotional and insomnia scores. ${ }^{15}$

The National Cancer Institute of Canada Clinical Trials Group conducted a phase II study evaluating OR to oral fludarabine in previously untreated CLL patients. Patients were treated with oral fludarabine $40 \mathrm{mg} / \mathrm{m}^{2} / \mathrm{d}$ for five days every 28 days for a maximum of six or eight cycles. One hundred twenty-six patients were eligible with a median age of 60.9 years. The OR was $64 \%$ with CR of $18 \%$ (NCI-WG criteria). Median PFS at a median follow up of 23.2 months was 15.3 months. Neutropenia grade 3-4 was observed in $51 \%$ of patients. The authors concluded that oral fludarabine is associated with response rates and a toxicity profile comparable to those of IV fludarabine given on a similar schedule. ${ }^{16}$

In 1999, a multicenter RCT (LRF CLL4) comparing fludarabine, fludarabine with cyclophosphamide (FC) and chlorambucil as front-line treatment in CLL was initiated. Fludarabine was given IV till February 2001 when the oral formulation became available. One hundred seven of 196 patients allocated to the fludarabine arm and 116/196 patients allocated to the FC arm received the oral formulation. Oral fludarabine as single-agent was given at a dose of $40 \mathrm{mg} / \mathrm{m}^{2} / \mathrm{d}$ for five days every 28 days for six cycles. The oral FC arm consisted of fludarabine $24 \mathrm{mg} / \mathrm{m}^{2} / \mathrm{d}$ plus cyclophosphamide $150 \mathrm{mg} / \mathrm{m}^{2} / \mathrm{d}$ each for five days every 28 days for six cycles. No difference in hematological and nonhematological toxicities was seen for oral and IV fludarabine. However responses seemed better for IV compared to oral therapy (Fludarabine: CR/Nodular PR [NPR] 54\% vs $41 \%$; FC: CR/NPR $73 \%$ vs $59 \%$ ). The authors of this study concluded that the observed difference in response was probable not due to the route of administration of fludarabine, but was more likely because of the inclusion of older patients with a poorer prognosis later in the study when all patients were guaranteed to receive oral therapy. The response rate also decreased by year of entry in the chlorambucil arm. ${ }^{17}$ 
Table I Results of clinical trials with fludarabine as single-agent or in combination given intravenously or orally

\begin{tabular}{lllllllll}
\hline References RCT & M & Median Route & Treatment regimen OR (\%) & CR (\%) & PFS (m) & Neutropenia (\%) & Infections (\%)
\end{tabular} age

Grade 3-4

Single-agent fludarabine in previously treated CLL

$\begin{array}{lllllllllll}\text { Keating }^{2} & \text { no } & 68 & 60 & \text { IV } & F 25-30 \mathrm{mg} / \mathrm{m}^{2} \times 5 / 28 \mathrm{~d} & 57 & 13 & 16(\mathrm{OS}) & 56 \text { (courses) } & \text { na } \\ \text { Boogaerts }^{13} & \text { no } & 78 & 63.4 & \text { oral } & F 40 \mathrm{mg} / \mathrm{m}^{2} \times 5 / 28 \mathrm{~d} & 51,3 & 17,9 & \text { na } & 53.8 & 7.7\end{array}$

Single-agent fludarabine in untreated CLL

\begin{tabular}{|c|c|c|c|c|c|c|c|c|c|c|}
\hline Leporrier $^{34}$ & yes & 341 & 62 & IV & $\mathrm{F} 25 \mathrm{mg} / \mathrm{m}^{2} \times 5 / 28 \mathrm{~d}$ & $7 I, I$ & 40,1 & 31.7 & 38 (courses?) & na \\
\hline $\mathrm{Rai}^{14}$ & yes & 179 & 64 & IV & $\mathrm{F} 25 \mathrm{mg} / \mathrm{m}^{2} \times 5 / 28 \mathrm{~d}$ & 63 & 20 & 20 & 27 & 16 \\
\hline Eichhorst $^{27}$ & yes & 164 & 59 & IV & $\mathrm{F} 25 \mathrm{mg} / \mathrm{m}^{2} \times 5 / 28 \mathrm{~d}$ & 83 & 7 & 20 & 26 & 8.7 \\
\hline Flinn 28 & yes & 137 & 61 & IV & $\mathrm{F} 25 \mathrm{mg} / \mathrm{m}^{2} \times 5 / 28 \mathrm{~d}$ & 59 & 7 & 19 & 63 & na \\
\hline Catovsky ${ }^{29}$ & yes & 196 & 64 & IV-oral & $\mathrm{F} 25(40) \mathrm{mg} / \mathrm{m}^{2} \times 5 / 28 d$ & 80 & 15 & 28 & na & na \\
\hline Rossi $^{15}$ & no & 81 & 61.2 & oral & $\mathrm{F} 40 \mathrm{mg} / \mathrm{m}^{2} \times 5 / 28 \mathrm{~d}$ & 80,2 & 12,3 & 27 & 32.1 & 4.9 \\
\hline Shustik ${ }^{16}$ & no & 126 & 60.9 & oral & $\mathrm{F} 40 \mathrm{mg} / \mathrm{m}^{2} \times 5 / 28 \mathrm{~d}$ & 64 & 18 & 15.3 & 51 & na \\
\hline \multicolumn{11}{|c|}{ Fludarabine plus cyclophosphamide in treated CLL } \\
\hline Wierda $^{21}$ & no & 111 & 59 & IV & $\begin{array}{l}\mathrm{F} 30 \mathrm{mg} / \mathrm{m}^{2} \times 3 / 28 \mathrm{~d}+ \\
\text { C } 300 \mathrm{mg} / \mathrm{m}^{2} \times 3 / 28 \mathrm{~d}\end{array}$ & 67 & 12 & 36 & 47 (courses) & na \\
\hline Robak $^{22}$ & yes & 276 & 63 & IV & $\begin{array}{l}\mathrm{F} 25 \mathrm{mg} / \mathrm{m}^{2} \times 3 / 28 \mathrm{~d}+ \\
\text { C } 250 \mathrm{mg} / \mathrm{m}^{2} \times 3 / 28 \mathrm{~d}\end{array}$ & 58 & 13 & 20.6 & 40 & 19 \\
\hline Forconi ${ }^{32}$ & no & 12 & $\geq 65$ & oral & $\begin{array}{l}\text { F } 25 \mathrm{mg} / \mathrm{m}^{2} \times 4 / 28 d+ \\
\text { C } 120 \mathrm{mg} / \mathrm{m}^{2} \times 4 / 28 d\end{array}$ & 83,5 & 25 & 48 (EFS) & 25 & 16.6 \\
\hline
\end{tabular}

Fludarabine plus cyclophosphamide in untreated CLL

\begin{tabular}{|c|c|c|c|c|c|c|c|c|c|c|}
\hline Eichhorst $^{27}$ & yes & 164 & 58 & IV & $\begin{array}{l}\mathrm{F} 25 \mathrm{mg} / \mathrm{m}^{2} \times 3 / 28 \mathrm{~d}+ \\
\text { C } 250 \mathrm{mg} / \mathrm{m}^{2} \times 3 / 28 \mathrm{~d}\end{array}$ & 94 & 24 & 48 & 55.5 & 8.7 \\
\hline Flinn ${ }^{28}$ & yes & $14 \mid$ & 61 & IV & $\begin{array}{l}\mathrm{F} 20 \mathrm{mg} / \mathrm{m}^{2} \times 5 / 28 \mathrm{~d}+ \\
\text { C } 600 \mathrm{mg} / \mathrm{m}^{2} / 28 \mathrm{~d}\end{array}$ & 74 & 23 & 32 & 69 & na \\
\hline Catovsky $^{29}$ & yes & 196 & 65 & IV-oral & $\begin{array}{l}\mathrm{F} 25(24) \mathrm{mg} / \mathrm{m}^{2} \\
\times 3(5) / 28 \mathrm{~d}+\mathrm{C} 250 \\
(120) \mathrm{mg} / \mathrm{m}^{2} \times 3(5) / 28 \mathrm{~d}\end{array}$ & 95 & 38 & 43 & na & na \\
\hline Hallek $^{30}$ & yes & 409 & 61 & IV & $\begin{array}{l}\mathrm{F} 25 \mathrm{mg} / \mathrm{m}^{2} \times 3 / 28 \mathrm{~d}+ \\
\text { C } 250 \mathrm{mg} / \mathrm{m}^{2} \times 3 / 28 \mathrm{~d}\end{array}$ & 95 & 27 & 32 & 20,9 & 14.8 \\
\hline Cazin ${ }^{31}$ & no & 75 & 57 & oral & $\begin{array}{l}\mathrm{F} 30 \mathrm{mg} / \mathrm{m}^{2} \times 5 / 28 \mathrm{~d}+ \\
\text { C } 200 \mathrm{mg} / \mathrm{m}^{2} \times 5 / 28 \mathrm{~d}\end{array}$ & 80 & 53 & 60 & 52 & 16 \\
\hline Forconi ${ }^{32}$ & no & 14 & $\geq 65$ & oral & $\begin{array}{l}\mathrm{F} 25 \mathrm{mg} / \mathrm{m}^{2} \times 4 / 28 \mathrm{~d}+ \\
\text { C } 120 \mathrm{mg} / \mathrm{m}^{2} \times 4 / 28 \mathrm{~d}\end{array}$ & 100 & 61.5 & 48 (EFS) & 21 & 0 \\
\hline Laurenti ${ }^{33}$ & no & 35 & 68 & oral & $\begin{array}{l}\mathrm{F} 30 \mathrm{mg} / \mathrm{m}^{2} \times 3 / 28 \mathrm{~d}+ \\
\text { C } 250 \mathrm{mg} / \mathrm{m}^{2} \times 3 / 28 \mathrm{~d}\end{array}$ & 77 & 40 & 23 & 45.7 & 0 \\
\hline \multicolumn{11}{|c|}{ Fludarabine plus alemtuzumab in treated CLL } \\
\hline Elter ${ }^{23}$ & no & 36 & 61.4 & IV & $\begin{array}{l}\mathrm{F} 30 \mathrm{mg} / \mathrm{m}^{2} \times 3 / 28 \mathrm{~d}+ \\
\text { A } 30 \mathrm{mg} \times 3 / 28 \mathrm{~d}\end{array}$ & 83 & 30 & $35.6(\mathrm{OS})$ & 26 (courses) & 5 (courses) \\
\hline Hwang ${ }^{24}$ & no & 5 & 72 & oral-sc & $\begin{array}{l}\text { F } 40 \mathrm{mg} / \mathrm{m}^{2} \times 5 / 28 \mathrm{~d}+ \\
\text { A } 30 \mathrm{mg} / \mathrm{dl}-3-5 / 28 \mathrm{~d}\end{array}$ & 100 & 60 & na & na & na \\
\hline \multicolumn{11}{|c|}{ Fludarabine-cyclophosphamide plus alemtuzumab in treated CLL } \\
\hline Elter 25 & no & 55 & 63 & IV-sc & $\begin{array}{l}\text { F } 25 \mathrm{mg} / \mathrm{m}^{2} \times 3+ \\
\text { C } 200 \mathrm{mg} / \mathrm{m}^{2} \times 3+ \\
\text { A } 30 \mathrm{mg} \times 3 / 28 \mathrm{~d}\end{array}$ & 83 & 38 & na & na & na \\
\hline Montillo26 & no & 25 & 57 & oral-sc & $\begin{array}{l}\mathrm{F} 40 \mathrm{mg} / \mathrm{m}^{2} \times 3+ \\
\text { C } 250 \mathrm{mg} / \mathrm{m}^{2} \times 3+ \\
\text { A } 10-20 \mathrm{mg} \times 3 / 28 \mathrm{~d}\end{array}$ & 79 & 37 & na & 43 (courses) & na \\
\hline
\end{tabular}

Abbreviations: C, cyclophosphamide; CR, complete response; EFS, event free survival; F, fludarabine; Infections (\%), \% of patients treated; n, number of evaluable patients; na, not available; Neutropenia (\%), \% of patients treated; OR, overall response; OS, overall survival; PFS(m), progression free survival (months); RCT, randomized control trial; sc, subcutaneous. 


\section{Fludarabine combination therapy}

A variety of agents has been combined with fludarabine in an attempt to improve efficacy. Prednisone or chlorambucil in addition to fludarabine increased hematological toxicity and the incidence of infection without improving response rates. ${ }^{14,18}$ In vitro and in vivo data have shown synergy between fludarabine and DNA-damaging agents (cyclophosphamide, mitoxantrone, cisplatinum, etc). DNA interstrand cross-links induced by cyclophosphamide can persist because DNA repair enzymes are inhibited by fludarabine. Cyclophosphamide also enhances the incorporation of F-ara-AMP in DNA, enhancing cell death. ${ }^{19}$ Synergy between fludarabine and monoclonal antibodies (rituximab, alemtuzumab) has also been shown. Rituximab downregulates antiapoptotic proteins while fludarabine downregulates anticomplement proteins, making cells more vulnerable to apoptosis ${ }^{20}$ The most thoroughly studied combinations are FC and FC plus rituximab (FCR) IV. Other effective combinations are $\mathrm{FC}$ or FCR plus mitoxantrone (FCM, FCM-R) and $\mathrm{F}$, FC or FCR plus alemtuzumab (F-Cam, FC-Cam, CFAR).

\section{Fludarabine combination in previously treated patients}

One hundred eleven CLL patients with recurrent/refractory disease were treated at a single center with fludarabine $30 \mathrm{mg} / \mathrm{m}^{2}$ and cyclophosphamide $300 \mathrm{mg} / \mathrm{m}^{2}$ IV for three days, every four weeks for a total of six planned cycles. Median age was 59 years (range 31-79 years). OR was $67 \%$ with a CR of $12 \%$ (NCI-WG criteria). The estimated time to disease progression for responders was 36 months. Median OS for all patients was 31 months and exceeded 71 months for the complete responders. Grade 3-4 neutropenia was observed in $47 \%$ of treatment cycles. ${ }^{21}$

The rituximab in the study of relapsed chronic lymphocytic leukemia (REACH) trial compared FC with FCR IV in previously treated CLL patients. 552 patients with a median age of 63 years were randomized. OR (70\% vs $58 \%)$ and CR (24\% vs $13 \%)$ (NCI-WG criteria) was better for FCR. Median PFS improved from 20.6 to 30.6 months. Grade 3-4 neutropenia was comparable ( $42 \%$ vs $40 \%$ ) as was infection rate $(18 \%$ vs $19 \%){ }^{22}$

A phase II study determined the efficacy and safety of fludarabine $30 \mathrm{mg} / \mathrm{m}^{2}$ and alemtuzumab $30 \mathrm{mg}$ IV administered on three consecutive days, every 28 days for up to six cycles in CLL patients with relapsing/refractory disease. Thirty-six patients with a median age of 61.4 years (range $38-80$ years) were treated. OR reached $83 \%$ with $30 \%$ CR (NCI-WG criteria). Grade 3-4 neutropenia and infections were seen in $26 \%$ and $5 \%$ of treatment cycles. Median OS was 35.6 months. ${ }^{23}$

A pilot study assessed the safety and efficacy of oral fludarabine $40 \mathrm{mg} / \mathrm{m}^{2}$ for five days and subcutaneous (sc) alemtuzumab $30 \mathrm{mg}$ on days 1,3 , and 5 every four weeks for a total of two to six cycles in the treatment of five relapsed/refractory CLL patients. Median patient age was 72 years (range 60-81 years). All patients responded (CR, 3; $\mathrm{PR}, 2)$. The authors concluded that this regimen constituted a well-tolerated, self-administered, outpatient-based treatment in elderly patients. ${ }^{24}$

The combination of fludarabine $\left(25 \mathrm{mg} / \mathrm{m}^{2} \mathrm{IV}\right)$, cyclophosphamide $\left(200 \mathrm{mg} / \mathrm{m}^{2} \mathrm{IV}\right)$ and alemtuzumab (30 mg sc) was tested in a phase II trial. Fifty-five patients with relapsing/refractory CLL were included. Twenty-four patients were evaluable for response and safety. Median age was 63 years (range $47-78$ years). OR was $83 \%$ with CR of $38 \% .{ }^{25}$

Another phase II trial enrolled 25 patients with relapsing/ refractory CLL. The treatment regimen consisted of fludarabine $40 \mathrm{mg} / \mathrm{m}^{2}$ and cyclophosphamide $250 \mathrm{mg} / \mathrm{m}^{2}$ given orally for three days and alemtuzumab 10 to $20 \mathrm{mg}$ given $\mathrm{sc}$ for three days. Cycles were repeated every 28 days for a maximum of six cycles. Median age was 57 years (range $42-79$ years). Grade 3-4 neutropenia was seen in $43 \%$ of administered courses. Four major infections were mentioned. OR was $79 \%$ with CR $37 \%{ }^{26}$

\section{Fludarabine combination}

\section{in untreated patients}

Three RCTs compared fludarabine with FC in untreated CLL patients. FC chemotherapy resulted in a higher CR and OR ( $23.4 \%$ to $38 \%$ and $74.3 \%$ to $95 \%$, respectively) compared with fludarabine alone ( $4.6 \%$ to $15 \%$ and $59.5 \%$ to $83 \%$, respectively). FC also resulted in a longer PFS. So far, no difference in OS has been seen. Grade 3-4 neutropenia was increased although the rate of severe infections was comparable in both study arms of all three studies. ${ }^{27-29}$

The German CLL study group (GCLLSG) CLL8 trial compared FC versus FCR IV as front-line treatment in CLL. Eight hundred seventeen patients with a cumulative illness rating scale (CIRS) score of up to 6 and a creatinine clearance $(\mathrm{CrCl})>70 \mathrm{ml} / \mathrm{min}$ were randomized. The median age was 61 years (range 30-81 years). The addition of rituximab almost doubled the CR rate from $27 \%$ to $52 \%$ and improved OR to $95 \%$. Median PFS was 42.8 months for the FCR group versus 32 months for the FC group. Grade 3-4 neutropenia increased from $20.9 \%$ to $33.6 \%$. Grade $3-4$ infection rate remained comparable (14.8\% versus $18.8 \%) .{ }^{30}$ 
A multicenter single-arm study tested the efficacy and toxicity of the oral combination of fludarabine $\left(30 \mathrm{mg} / \mathrm{m}^{2}\right)$ and cyclophosphamide $\left(200 \mathrm{mg} / \mathrm{m}^{2}\right)$ for five days every 28 days for six cycles in 75 untreated CLL patients. The median age of the patients at the time of inclusion was 57 years (37-66 years). Oral FC demonstrated high efficacy with OR and CR of $80 \%$ and $53 \%$, respectively. Median OS and median treatment free interval had not been reached after seven years of follow-up. Median PFS was five years. Toxicity was acceptable with grade 3-4 neutropenia and infections seen in $52 \%$ and $16 \%$ of treatment cycles. Gastrointestinal toxicity was generally limited to grade $1-2$. Nausea grade 3-4 was mentioned in $2 \%$ of treatment cycles while vomiting and diarrhea grade 3-4 was mentioned in $0.27 \%$ and $0.2 \%$ of cycles, respectively. Antiemetic prophylaxis was recommended to avoid lesser drug absorption due to vomiting. The authors concluded that compliance to the oral formulation was excellent. ${ }^{31}$

Efficacy and toxicity of oral FC at reduced doses (fludarabine $25 \mathrm{mg} / \mathrm{m}^{2}(\max 40 \mathrm{mg} / \mathrm{d}$ ) d1-4 and cyclophosphamide $120 \mathrm{mg} / \mathrm{m}^{2}$ (max $200 \mathrm{mg} / \mathrm{d}$ ) d1-4 every four weeks for a maximum of four cycles) in elderly patients ( $>65$ years) with CLL was tested. OR in the 14 previously untreated patients was $100 \%$ with a CR of $61.5 \%$ and event-free survival (EFS) at 24 months of $71 \%$. In the 12 previously treated patients an OR of $83.5 \%$ with CR of $25 \%$ was seen with an EFS at 24 months of $83 \%$. No patients reported nausea, vomiting, or required antiemetic treatment. Grade 2 diarrhea was reported in two patients. ${ }^{32}$

Thirty-seven patients with previously untreated CLL received oral fludarabine $\left(30 \mathrm{mg} / \mathrm{m}^{2}\right)$ and cyclophosphamide $\left(250 \mathrm{mg} / \mathrm{m}^{2}\right) \mathrm{d} 1-3$ every four weeks for six cycles. Among the 35 evaluable patients, OR was $77 \%$ with CR of $40 \%$. The median PFS was 23 months and median time to retreatment was 38 months. Grade 3-4 neutropenia was observed in 16 patients. Gastrointestinal toxicity was mild. Nausea grade 1-2 was seen in 12 patients and grade 3 in two patients. Vomiting grade 1-2 was seen in six patients with one patient reporting grade 3 toxicity. ${ }^{33}$

\section{Tolerability \\ Hematological toxicity}

Fludarabine suppresses severely bone marrow function with induction of neutropenia, thrombocytopenia and anemia. Myelosuppression is the major dose-limiting adverse effect. In a RCT conducted by the French Cooperative Group on CLL fludarabine IV induces grade 3-4 neutropenia, thrombocytopenia, and anemia in $38 \%, 15 \%$, and $18 \%$ of previously untreated CLL patients, respectively. ${ }^{34}$ This myelotoxicity is even more pronounced when patients with recurrent of refractory disease are treated with fludarabine. Neutropenia and thrombocytopenia were observed in $56 \%$ and $25 \%$ of evaluable courses, respectively. ${ }^{2}$ Using the oral formulation, grade 3-4 neutropenia, thrombocytopenia, and anemia developed in $32.1 \%, 4.9 \%$, and $9.9 \%$ of previously untreated and $53.8 \%, 25.6 \%$, and $4.4 \%$ of previously treated CLL patients, respectively. ${ }^{13,15}$ Data from two RCT's combining FC with or without rituximab were recently presented. With front-line treatment grade 3-4 neutropenia, thrombocytopenia and anemia were observed in $21 \%, 11 \%$, and $7 \%$ of the $\mathrm{FC}$ group and in $34 \%, 7 \%$, and $5 \%$ of the FCR group, respectively. ${ }^{30}$ In patients previously treated, grade 3-4 neutropenia, thrombocytopenia, and anemia were seen in $40 \%, 9 \%$, and $5 \%$ of patients treated with $\mathrm{FC}$ and in $42 \%, 11 \%$, and $2 \%$ of patients treated with FCR, respectively. ${ }^{22}$ Prolonged cytopenia after fludarabine-based combination regimens have been observed. It can take several months before resolution is complete although recurrent cytopenic episodes can occur in the first year of remission..$^{35,36}$ Fludarabine given as first line or salvage treatment frequently reduces CD4 and CD8 cells to less than $200 / \mu 1$ during the treatment period and this suppression can persist for more than one year. ${ }^{18,37}$

\section{Infectious complications}

Infection in CLL is multifactorial. The major risk factors are immune defects inherent to the primary disease process and therapy related immunosuppression. Fludarabine is not only linked to a higher risk of infections than alkylatingagent-based treatment, it changes also the spectrum of infections seen in these patients. In addition to bacterial infections common to patients with CLL, opportunistic infections (Listeria, mycobacterial species, Nocardia, Candida, Aspergillus, Cryptococcus, Pneumocystis, and herpesviruses) become more frequent. Although the value of routine antiviral and pneumocystis prophylaxis in fludarabine treated patients is not examined prospectively, a lot of clinicians recommend routine antibacterial and antiviral prophylaxis during and after PA treatment. ${ }^{38}$ Myeloid growth factor support can be used according to American Society of Clinical Oncology guidelines to reduce myelosuppression as well to allow the delivery of full-dose therapy. ${ }^{39}$ Recently, when reviewing the data of the GCLLSG CLL4 trial, it was suggested that routine antibiotic or virostatic prophylaxis and pre-emptive treatment with G-CSF is not necessary in first-line therapy with fludarabine-based regimens in CLL patients younger than 66 years. ${ }^{40}$ 
Guidelines for the use of intravenous immunoglobulin (IVIG) replacement in CLL do not exist. Most clinicians find it justifiable to use IVIG in CLL patients with hypogammaglobulinemia and recurrent bacterial infections. ${ }^{41}$

CLL patients are not only at risk for acquiring infections but are also at risk for reactivation of latent infections (herpesviruses, Toxoplasma, Hepatitis B virus, JC virus). ${ }^{42,43}$ Progressive multifocal leukoencephalopathy (PML) is a subacute demyelinating disease caused by infection of oligodendrocytes with the JC virus. This neurological disorder is well known in AIDS patients but is diagnosed more frequently also among patients with hematological malignancies, chronic inflammatory diseases and after organ transplantantion due to the use of immunosuppressive agents. Recently there were concerns about the potential for PML among rituximab-treated patients. However, 26/52 reported cases were also treated with PA in the past. ${ }^{44}$ One case of PML developing in a CLL patient after treatment with oral fludarabine has been published. This patient developed symptoms seven months after completion of treatment when T-lymphocytes were still very low. ${ }^{45}$

The combination of fludarabine plus corticosteroids and also fludarabine plus chlorambucil must be discouraged because the combination significantly increased the severity and incidence of infections compared with fludarabine monotherapy. ${ }^{46,47}$

\section{Neurotoxicity}

Dose dependent neurotoxicity has been observed with fludarabine. The high doses of fludarabine $\left(>96 \mathrm{mg} / \mathrm{m}^{2} / \mathrm{d}\right.$ for 5-7 days) used in the treatment of acute leukemia were associated with severe late onset neurotoxicity in $36 \%$ of cases. The clinical symptoms consisted of altered mental status, seizures, paraparesis, progressive encephalopathy and coma. ${ }^{48}$ Ocular toxicity, although infrequent, may be rapidly sight-threatening and largely irreversible. ${ }^{49}$ With conventional dose fludarabine neurotoxicity is mild and reversible in the majority of cases. In reviewing 2,136 patients treated with fludarabine for hematological malignancies, 336 patients (16\%) reported some degree of neurotoxicity. Reversible neurological events included seizures, loss of consciousness, blurred vision, and leg weakness. ${ }^{50}$ Grade 3 ocular toxicity (subtotal loss of vision) and grade 4 (blindness) was reported in $1 \%$ and $0.3 \%$ of patients followed by the group $\mathrm{C}$ protocol mechanism of the national cancer institute..$^{51} \mathrm{PML}$ must be excluded when neurological symptoms occur. As there is no known prophylaxis or treatment for neurotoxicity except discontinuing fludarabine immediately, a heightened awareness of the neurological vulnerability of some patients to standard doses of fludarabine is necessitated with the increasing use of this PA. In the pivotal trial conducted with oral fludarabine, one patient had a severe impairment of consciousness during the last treatment cycle. Mild consciousness-related adverse events were experienced by $15.4 \%$ of the other patients and $6.4 \%$ experienced mild to moderate peripheral neuropathy. ${ }^{13}$

\section{Autoimmune complications}

Autoimmune phenomena like autoimmune hemolytic anemia (AIHA), autoimmune thrombocytopenia, thrombocytopenic purpura, pemphigus, acquired hemophilia, and Evans' syndrome have all been described in CLL. AIHA is the most common autoimmune disorder. An incidence of AIHA of $8.6 \%$ in untreated patients and $11 \%$ after treatment (mainly with alkylating agents) was seen in the MRC CLL trials in the last 20 years..$^{52}$ In the front-line LRF CLL4 trial, AIHA was diagnosed equally in the chlorambucil and fludarabine patient groups $(12 \%-11 \%)$ and was less common in patients treated with FC in combination (5\%) suggesting a preventive effect. ${ }^{29}$ In the context of heavily pretreated CLL, fludarabine is thought to predispose to AIHA. However, in the context of previously untreated CLL, fludarabine seems no more hemolytic than other agents. AIHA can occur in patients with or without a previous history of AIHA, with or without a Coombs-positive result and whether in remission from their CLL or not. Hemolysis after fludarabine could be life threatening and some fatalities have been reported. Rechallenge with fludarabine after AIHA should be avoided because the majority of patients will develop a recurrence of hemolysis.

\section{Gastrointestinal toxicity}

In a RCT comparing fludarabine IV and cyclophosphamide, doxorubicin and prednisone grade 1-2 nausea/vomiting and diarrhea was reported respectively in $5 \%$ and $4 \%$ of patients treated with fludarabine. ${ }^{3}$ With the oral formulation, grade 1-2 nausea/vomiting was reported in $37 \%$ to $38.2 \%$ with grade $3-4$ toxicity noticed in $1.2 \%$ to $1.3 \%$ of patients. Grade 1-2 diarrhea was reported in 34.6\% to $42 \%$ of patients with $3.8 \%$ to $6.2 \%$ suffering from grade 3-4 toxicity. ${ }^{13,15}$ Gastrointestinal toxicity is of particular importance in the assessment of any oral formulation since nausea, vomiting, and diarrhea make the drug not only unacceptable for the patient but also affect the absorption of the drug. 


\section{Second malignancies}

Prolonged immunosuppression may increase the risk of second malignancies. A retrospective analysis has been done in 724 CLL patients treated with fludarabine. No increased risk was found for second neoplasms already associated with a diagnosis of CLL. ${ }^{3}$

Myelodysplasia and secondary acute myeloid leukemia are rarely reported in CLL patients treated with fludarabine monotherapy. However the combination of fludarabine with DNA-damaging agents may increase this risk up to $10 \%{ }^{54}$ Transformation of CLL to large cell lymphoma or Hodgkin's disease is known as Richter's syndrome (RS). According to the literature, $1 \%$ to $10 \%$ of CLL patients develop this high-grade malignancy. The relationship between the immunosuppressive effect of PA and monoclonal antibodies in the development of this large cell transformation remains a controversial issue. In reviewing the literature we were not able to show a statistically significant difference in RS incidence between patients treated with and without PA. We suppose that the profound and early occurring T cell depletion could be responsible for RS soon after the start of and during treatment whereas the prolonged immunosuppression can explain RS later on even when complete remission is sustained. ${ }^{55}$ Some of these lymphoproliferations were clearly Epstein-Barr virus (EBV)-mediated. ${ }^{56}$ Recently it was suggested that aggressive lymphomas developing after T-cell-depleting therapies should be seen as a novel type of immunodeficiency-related lymphoma and not as RS. ${ }^{57}$

\section{Tumor lysis syndrome}

Tumor lysis syndrome (TLS) is a rare complication of fludarabine treatment. Clinical and laboratory features consistent with TLS were present in only 20 of 6,137 patients treated with fludarabine $(0.33 \%)$. After intravenous infusion TLS developed approximately on day 7. Patients with a high tumor burden, high rate of proliferation, and disease highly responsive to therapy are at risk for TLS. ${ }^{58}$ TLS can be easily prevented with allopurinol prophylaxis and the encouragement of hydration with oral fluids during treatment. ${ }^{59}$ Two case reports describing TLS after oral fludarabine were published. ${ }^{60,61}$ One of these patients developed TLS during the two first treatment cycles. TLS was diagnosed two weeks after the oral administration, later than what is seen after IV fludarabine. ${ }^{61}$

\section{Transfusion and vaccination policy}

A small number of transfusion-associated graft-versus-host disease was noted after fludarabine treatment. This led to the recommendation that fludarabine-treated patients should receive irradiated blood products if they require transfusion. ${ }^{62}$ There is no international guideline about the duration of this recommendation. A lot of clinicians maintain this precaution for at least a year but additional research is required to determine whether such a transfusion policy is required during the patient's whole lifetime.

It has been shown that immune responses to vaccination in CLL patients are suboptimal due to impaired antibody production as well as to defects in antigen presentation. ${ }^{41}$ Immunocompetence is further altered by chemotherapy through depletion of B- and T-lymphocytes, plasma cells, and natural killer cells. Vaccine recommendations specific for CLL patients are lacking. However pneumococcal and influenza vaccines are advocated for adult patients with altered immunocompetence on the basis of proven effectiveness and an increased risk for infections if these vaccines are withheld. Vaccination or revaccination with inactivated vaccines should be administered at least three months after the end of immunosuppressive treatment if immunocompetence is restored. ${ }^{63}$ Evaluation of immune recovery should not only include measurement of lymphocyte subsets and immunoglobulin levels, but also T-lymphocyte proliferation in response to specific or nonspecific stimuli. ${ }^{64}$ Until specific scientific data about vaccination in CLL after fludarabinebased treatment become available and also knowing that immune reconstitution after these regimens is slow, it is safe to use the guidelines for revaccination of recipients of hematopoietic stem cell transplant. This means that inactivated influenza vaccine can be administered at least six months after the end of a PA-based regimen, while all other inactivated vaccines should begin 12 months after completion of these treatments. Vaccination with live vaccines should be avoided during treatment with fludarabine and 24 months after treatment. ${ }^{63}$

\section{Dosage and administration and dose adjustment}

For the treatment of CLL the recommended IV dose of fludarabine as a single-agent is $25 \mathrm{mg} / \mathrm{m}^{2}$ daily for five days and repeated at 28-day intervals, administered as a 30-minute intravenous infusion or as an intravenous bolus injection. Oral fludarabine is given at a dosage of $40 \mathrm{mg} / \mathrm{m}^{2}$ once daily for five days, repeated every four weeks for up to six cycles. The IV combination tested most extensively in phase III trials is fludarabine $25 \mathrm{mg} / \mathrm{m}^{2}$ and cyclophosphamide $250 \mathrm{mg} / \mathrm{m}^{2}$ daily for three days repeated every 28 days for six cycles. This is comparable to an oral combination of 
fludarabine $40 \mathrm{mg} / \mathrm{m}^{2}$ and cyclophosphamide $250 \mathrm{mg} / \mathrm{m}^{2}$ daily for three days or fludarabine $24 \mathrm{mg} / \mathrm{m}^{2}$ and cyclophosphamide $150 \mathrm{mg} / \mathrm{m}^{2}$ daily for five days repeated every 28 days for six cycles. The fludarabine tablets can be taken either on an empty stomach or with food, and must be swallowed whole with water. They should not be chewed upon or crushed. One film-coated tablet contains $10 \mathrm{mg}$ fludarabine. The tablets must be stored between $15^{\circ} \mathrm{C}$ and $30^{\circ} \mathrm{C}$.

Fludarabine is eliminated primarily through renal excretion. Until recently the product monograph of fludarabine recommended a dosage reduction of up to $50 \%$ for patients with a $\mathrm{CrCl}$ of 30 to $70 \mathrm{ml} / \mathrm{min}$. Fludarabine was even contraindicated in patients with a $\mathrm{CrCl}<30$ $\mathrm{ml} / \mathrm{min} .{ }^{65}$ With the FDA approval of oral fludarabine at the end of 2008, a revised product monograph was released recommending the following dose adjustments: reduce dose by $20 \%$ in patients with mild to moderate renal impairment $\left(\mathrm{CrCl} 30-70 \mathrm{ml} / \mathrm{min} / 1.73 \mathrm{~m}^{2}\right)$ and by $50 \%$ in patients with severe renal impairment $\left(\mathrm{CrCl}<30 \mathrm{ml} / \mathrm{min} / 1.73 \mathrm{~m}^{2}\right) .{ }^{66}$ Already in previous years these dose adjustments had been shown to result in similar drug AUC (0-24 hours) compared to patients with normal renal function receiving the standard recommended dose ${ }^{67}$ Careful monitoring of elderly patients ( $>75$ years) receiving fludarabine is recommended because data in this age group are limited.

\section{Warnings and precautions}

Myelosuppression is the major adverse event seen with fludarabine treatment. Careful hematologic monitoring is required.

Patients treated with fludarabine appear to be at an increased risk of infections, particularly opportunistic ones. The need for prophylactic antibiotic and virostatic treatment must be judged individually.

Fludarabine is relatively contraindicated in patients with active autoimmune hemolysis. Monitoring for hemolysis during treatment with fludarabine is warranted. Rechallenge with PA after fludarabine-related AIHA should be avoided.

Fludarabine must be discontinued immediately when neurological symptoms appear.

TLS must be prevented with allopurinol prophylaxis and the encouragement of oral hydration especially in patients with a high tumor burden.

Fludarabine-treated patients should receive irradiated blood products for at least one year and avoid vaccination with live vaccines for at least two years after the end of treatment.
$\mathrm{CrCl}$ should be measured at the start of every treatment cycle. Dose adjustments must be made according to the grade of reduced renal function.

It is recommended that men and women of childbearing potential take contraceptive measures during and for at least six months after the cessation of fludarabine because possible adverse effects on human fertility have not been adequately evaluated.

\section{Convenience and compliance}

In September 2001, NICE recommended oral fludarabine in preference to the intravenous formulation. The benefits of oral fludarabine include ease of administration with no need for repetitive venous punctures or indwelling catheters, absence of infusion-related adverse events such as extravasation, thrombosis, and catheter-related infections, reduced need for transportation and travel stress, which could improve the quality of life of patients. In addition medical costs should be reduced because of fewer hospital visits and fewer health care worker interventions. ${ }^{68}$

Although the use of oral fludarabine seems more convenient than the IV formulation, we must be aware of the compliance behavior. A patient is optimally adherent if no doses are missed, no extra doses are taken, and no doses are taken in the wrong quantity or at the wrong time. A patient has an optimal persistence if he or she takes a medication as long as it is prescribed. Adherence should never be assumed even in the treatment of hematologic malignancies. Patients must be aware that suboptimal adherence may prove to be the greatest barrier to the effective use of oral agents. ${ }^{69}$

Approximately two thirds of patients with CLL or indolent B-cell lymphoma are aged over 60 years. Especially in this elderly population the following factors must be taken into consideration when prescribing oral chemotherapy: age related physiological changes affecting clinical pharmacology (decreased absorption, altered distribution and metabolism, reduced renal clearance), polypharmacy, the patient's capacity to self-administer medication and safety concerns for the older patient and his or her carers. ${ }^{6}$

\section{Conclusion}

CLL combination treatment based on PAs such as fludarabine is associated with higher response rates and much longer remissions than traditional therapies such as chlorambucil. Patients treated with current therapies also seem to have a better OS compared to those in historical series. Nevertheless, until now no RCT has been able to show a survival benefit. 
Fludarabine is the only PA with an available oral formulation. No RCT is available to formally prove noninferiority of oral to IV fludarabine. The efficacy data retrieved from observational studies with oral fludarabine as singleagent or in combination with cyclophosphamide seem very similar to the historical data concerning treatment with IV fludarabine. Myelosuppression and infectious complications remain the most common adverse events and seem to be independent of the administration route. Gastrointestinal adverse effects however seem to occur more commonly with the oral formulation than previously reported with the IV formulation. Nevertheless most adverse events were mild to moderate in severity with no drug withdrawals reported.

Oral fludarabine eliminates the need for repetitive venous access and its potential complications and reduces the visits to the outpatient clinic which means less need for transportation and less travel stress. Medical costs should be reduced because of fewer hospital visits and fewer health care worker interventions. However, in some countries the cost of oral anti-cancer agents is much higher than for the parenteral counterpart due to reimbursement issues, making the oral treatment less appealing.

Treatment adherence is probably not a major issue because oral fludarabine is given only three to four days a month and can be taken once daily without diet restrictions. Compliance can further be improved by optimizing communication between the patient and health care workers with adequate education about the treatment schedule and possible adverse events.

Oral anticancer drugs are believed to suit elderly patients especially. According to data from the Surveillance, Epidemiology and End Results (SEER) Program of the United States National Cancer Institute, 31\% of CLL patients are aged between 65 and 74 years while $44 \%$ are 75 years or older. The LRF CLL4 trial has shown that more elderly patients were included if oral therapy could be guaranteed. Especially in this elderly population we must consider agerelated physiological changes, comorbidities, and polypharmacy that can influence the pharmacological properties of the oral drug.

Now that oral fludarabine is available in a large part of the world and more trials have confirmed efficacy and tolerability of the oral formulation, we believe that the IV formulation can be replaced by the oral drug when this is more convenient for the patient.

\section{Disclosures}

The authors report no conflicts of interest in this work.

\section{References}

1. CLL trialists' collaborative group. Chemotherapeutic options in chronic lymphocytic leukemia: a meta-analysis of the randomized trials. $J$ Natl Cancer Inst. 1999;91(10):861-868.

2. Keating MJ, Kantarjian H, Talpaz M, et al. Fludarabine: a new agent with major activity against chronic lymphocytic leukemia. Blood. 1989;74(1):19-25.

3. Johnson S, Smith AG, Löffler H, et al. Multicentre prospective randomised trial of fludarabine versus cyclophosphamide, doxorubicin, and prednisone (CAP) for treatment of advanced-stage chronic lymphocytic leukaemia. The French Cooperative Group on CLL. Lancet. 1996;347(9013):1432-1438.

4. Steurer M, Pall G, Richards S, et al. Single-agent purine analogues for the treatment of chronic lymphocytic leukemia: a systematic review and meta-analysis. Cancer Treat Rev. 2006;32(5):377-389.

5. Plunkett W, Gandhi V, Huang P, et al. Fludarabine: pharmacokinetics, mechanisms of action, and rationales for combination therapies. Semin Oncol. 1993;20(5 Suppl 7):S2-S12.

6. Huang P, Sandoval A, Van Den Neste E, Keating MJ, Plunkett W. Inhibition of RNA transcription: a biochemical mechanism of action against chronic lymphocytic leukemia cells by fludarabine. Leukemia. 2000;14(8):1503-1413.

7. Kemena A, Keating MJ, Plunkett W, et al. Plasma and cellular bioavailability of oral fludarabine [abstract]. Blood. 1991;78(Suppl):S52a.

8. Foran JM, Oscier D, Orchard J, et al. Pharmacokinetic study of single doses of oral fludarabine phosphate in patients with low-grade non-Hodgkin's lymphoma and B-cell chronic lymphocytic leukemia. J Clin Oncol. 1999;17(5):1574-1579.

9. Oscier D, Orchard JA, Culligan D, et al. The bioavailability of oral fludarabine phosphate is unaffected by food. Hematol J. 2001;2(5): 316-321.

10. Ogawa Y, Hotta T, Tobinai K, et al. Phase I and pharmacokinetic study of oral fludarabine phosphate in relapsed indolent B-cell non-Hodgkin's lymphoma. Ann Oncol. 2006;17(2):330-333.

11. Plosker GL, Figgitt DP. Oral fludarabine. Drugs. 2003;63(21): 2317-2323.

12. Cheson BD, Bennett JM, Grever M, et al. National cancer institutesponsored working group guidelines for chronic lymphocytic leukemia: revised guidelines for diagnosis and treatment. Blood. 1996;87(12):4990-4997.

13. Boogaerts MA, Van Hoof A, Catovsky D, et al. Activity of oral fludarabine phosphate in previously treated chronic lymphocytic leukemia. J Clin Oncol. 2001;19(22):4252-4258.

14. Rai KR, Peterson BL, Appelbaum FR, et al. Fludarabine compared with chlorambucil as primary therapy for chronic lymphocytic leukemia. N Eng J Med. 2000;343(24):1750-1757.

15. Rossi JF, Van Hoof A, de Boeck K, et al. Efficacy and safety of oral fludarabine phosphate in previously untreated patients with chronic lymphocytic leukemia. J Clin Oncol. 2004;22(7):1260-1267.

16. Shustik C, Turner R, Desjardin P, et al. A national cancer institute of Canada clinical trials group (NCIC CTG) phase II study of oral fludarabine in untreated B-cell chronic lymphocytic leukemia [abstract]. Blood. 2005;106(11):835a.

17. Hilmen P, Richards S, Catovsky D; UK NRCI CLL trials group. Comparison of oral and intravenous fludarabine in the LRF CLL4 trial [abstract]. Blood. 2005;106(11):214a.

18. O'Brien S, Kantarjian H, Beran M, et al. Results of fludarabine and prednisone therapy in 264 patients with chronic lymphocytic leukemia with multivariate analysis-derived prognostic model for response to treatment. Blood. 1993;82(6):1695-1700.

19. O'Brien S, Kantarjian H, Cortes J, et al. Results of the fludarabine and cyclophosphamide combination in chronic lymphocytic leukemia. J Clin Oncol. 2001;19(5):1414-1420.

20. Schulz H, Klein SK, Rehwald U, et al. Phase 2 study of a combined immunochemotherapy using rituximab and fludarabine in patients with chronic lymphocytic leukemia. Blood. 2002;100(9):3115-3120. 
21. Wierda W, O'Brien S, Faderl S, et al. A retrospective comparison of three sequential groups of patients with recurrent/refractory chronic lymphocytic leukemia treated with fludarabine-based regimens. Cancer. 2005;106(2):337-345.

22. Robak T, Moiseev SI, Dmoszynska A, et al. Rituximab, fludarabine and cyclophosphamide prolongs progression free survival in relapsed or refractory chronic lymphocytic leukemia compared with FC alone: final results from the international randomized phase III REACH trial [abstract]. Blood. 2008;112(11):3.

23. Elter T, Borchmann P, Schulz H, et al. Fludarabine in combination with alemtuzumab is effective and feasible in patients with relapsed or refractory B-cell chronic lymphocytic leukemia: results of a phase II trial. J Clin Oncol. 2005;23(28):7024-7031.

24. Hwang WY, Dearden C, Loh YS, et al. Outpatient-based therapy of oral fludarabine and subcutaneous alemtuzumab for Asian patients with relapsed/refractory chronic lymphocytic leukemia. Adv Hematol. 2009;DOI:10.1155/2009/547582.

25. Elter T, James R, Stilgenbauer S, Ritgen M, Hallek M, Engert A. Chemoimmuno-therapy with fludarabine, cyclophosphamide and alemtuzumab in patients with relapsed/refractory CLL: interim analysis of the CLL2L trial of the German CLL study group [abstract]. Blood. 2008;112(11):1087.

26. Montillo M, Miqueleiz S, Tedeschi A, et al. Combined fludarabine, cyclophosphamide and alemtuzumab (FCC), an active regimen for treated patients with chronic lymphocytic leukemia (CLL) [abstract]. Blood. 2007;110(11):922a.

27. Eichhorst BF, Bush R, Hopfinger G, et al. Fludarabine plus cyclophosphamide versus fludarabine alone in first-line therapy of younger patients with chronic lymphocytic leukemia. Blood. 2006;107(3):885-891.

28. Flinn IW, Neuberg DS, Grever MR, et al. Phase III trial of fludarabine plus cyclophosphamide compared with fludarabine for patients with previously untreated chronic lymphocytic leukemia: US intergroup trial E2997. J Clin Oncol. 2007;25(7):793-798.

29. Catovsky D, Richards S, Matutes E, et al. Assessment of fludarabine plus cyclophosphamide for patients with chronic lymphocytic leukemia (the LRF CLL4 trial): a randomized controlled trial. Lancet. 2007;370(9583):230-239.

30. Hallek M, Fingerle-Rowson G, Fink A, et al. Immunochemotherapy with fludarabine, cyclophosphamide and rituximab versus fludarabine and cyclophosphamide improves response rate and progression-free survival of previously untreated patients with advanced chronic lymphocytic leukemia [abstract]. Blood. 2008;112(11):125.

31. Cazin B, Divine M, Leprêtre S, et al. High efficacy with five days schedule of oral fludarabine phosphate and cyclophosphamide in patients with previously untreated chronic lymphocytic leukemia. $\mathrm{Br} J$ Haematol. 2008;143(1):54-59.

32. Forconi F, Fabbri A, Lenoci M, et al. Low-dose oral fludarabine plus cyclophosphamide in elderly patients with untreated and relapsed or refractory chronic lymphocytic leukemia. Hematol Oncol. 2008;26(4):247-251.

33. Laurenti L, Tarnani M, de Padua L, et al. Oral fludarabine and cyclophosphamide as front-line chemotherapy in patients with chronic lymphocytic leukemia. The impact of biological parameters in the response duration. Ann Hematol. 2008;87(11):891-898.

34. Leporrier M, Chevret S, Cazin B, et al. Randomized comparison of fludarabine, $\mathrm{CAP}$ and ChOP in 938 patients previously untreated stage $\mathrm{B}$ and $\mathrm{C}$ chronic lymphocytic leukemia patients. Blood. 2001;98(8):2319-2325.

35. Gill SI, Carney D, Ritchie DS, et al. The frequency, manifestations and duration of prolonged cytopenias after first-line fludarabinecombination chemotherapy for chronic lymphocytic leukemia and non-Hodgkin lymphoma [abstract]. Blood. 2008;112(11):1089.

36. Hamblin TJ. Fludarabine, cyclophosphamide and rituximab for chronic lymphocytic leukemia: no country for old men? Nat Clin Pract Oncol. 2009;6(3):130-131.

37. Keating MJ, O'Brien S, Lerner S, et al. Long-term follow-up of patients with chronic lymphocytic leukemia (CLL) receiving fludarabine regimens as initial therapy. Blood. 1998;92(4):1165-1171.
38. Samonis G, Kontoyiannis DP. Infectious complications of purine analog therapy. Curr Opin Infect Dis. 2001;14(4):409-413.

39. Smith TJ, Khatcheressian J, Lyman GH, et al. 2006 update of recommendations for the use of white blood cell growth factors: an evidence-based clinical practice guideline. J Clin Oncol. 2006;24(19):3187-3205.

40. Eichhorst BF, Bush R, Schweighofer C, et al. Due to low infection rates no routine anti-infective prophylaxis is required in younger patients with chronic lymphocytic leukaemia during fludarabine-based first line therapy. Br J Haematol. 2007;136(1):63-72.

41. Morrison VA. Management of infectious complications in patients with chronic lymphocytic leukemia. Hematology Am Soc Hematol Educ Program. 2007:332-338.

42. Bacchu S, Fegan C, Neal J. Cerebral toxoplasmosis in a patient with chronic lymphocytic leukemia treated with fludarabine. Br J Haematol. 2007;139(3):349.

43. Kiewe P, Seyfert S, Körper S, Rieger K, Thiel E, Knauf W. Progressive multifocal leukoencephalopathy with detection of JC virus in a patient with chronic lymphocytic leukemia parallel to onset of fludarabine therapy. Leuk Lymphoma. 2003;44(10):1815-1818.

44. Carson KR, Evens AM, Richey EA, et al. Progressive multifocal leukoencephalopathy after rituximab in HIV-negative patients: a report of 57 cases from the Research on Adverse Drugs Events and Reports project. Blood. 2009;113(20):4834-4840.

45. Cid J, Revilla A, Cervera A, et al. Progressive multifocal leukoencephalopathy following oral fludarabine for the treatment of chronic lymphocytic leukemia. Ann Hematol. 2000;79(7):392-395.

46. Anaissie EJ, Kontoyiannis DP, O'Brien S, et al. Infections in patients with chronic lymphocytic leukemia treated with fludarabine. Ann Intern Med. 1998;129(7):559-566.

47. Morrison VA, Rai KR, Bercedis L, et al. Impact of therapy with chlorambucil, fludarabine, or fludarabine plus chlorambucil on infections in patients with chronic lymphocytic leukemia: intergroup study cancer and leukemia B 9011. J Clin Oncol. 2001;19(16): 3611-3621.

48. Chun HG, Leyland-Jones BR, Caryk SM, Hoth DF. Central nervous system toxicity of fludarabine phosphate. Cancer Treat Rep. 1986; 70(10):1225-1228.

49. Ding X, Herzlich A, Bishop R, Tuo J, Chan C. Ocular toxicity of fludarabine: a purine analog. Expert Rev Ophtalmol. 2008;3(1):97-109.

50. Cheson BD, Vena DA, Foss FM, Sorensen JM. Neurotoxicity of purine analogs: a review. J Clin Oncol. 1994;12(10):2216-2228.

51. Sorensen JM, Vena DA, Fallavollita A, Chun HG, Cheson BD. Treatment of refractory chronic lymphocytic leukemia with fludarabine phosphate via the group $\mathrm{C}$ protocol of the National Cancer Institute: five-year follow-up report. J Clin Oncol. 1997;15(2):458-465.

52. Dearden C, Wade R, Else M, et al. The prognostic significance of a positive direct antiglobulin test in chronic lymphocytic leukemia: a beneficial effect of combination of fludarabine plus cyclophosphamide on the incidence of hemolytic anemia. Blood. 2008;111(4):1820-1826.

53. Cheson BD, Vena DA, Barrett J, Freidlin B. Second malignancies as a consequence of nucleoside analog therapy for chronic lymphoid leukemias. J Clin Oncol. 1999;17(8):2454-2460.

54. Tam CS, Seymour JF, Miles Prince H, et al. Treatment-related myelodysplasia following fludarabine combination chemotherapy. Haematologica. 2006;91(11):1546-1550.

55. Janssens A, Berth M, De Paepe P, et al. EBV negative Richter's syndrome from a coexistent clone after salvage treatment with alemtuzumab in a CLL patient. Am J Hematol. 2006;81(9):706-712.

56. Thornton PD, Bellas C, Santon A, et al. Richter's transformation of chronic lymphocytic leukemia. The possible role of fludarabine and the Epstein-Barr virus in its pathogenesis. Leuk Res. 2004;29(4): 389-395.

57. Rossi D, Gaidano G. Richter syndrome: molecular insights and clinical perspectives. Hematol Oncol. 2009;27(1):1-10.

58. Cheson BD, Frame JN, Vena D, Quashu N, Sorensen JM. Tumor lysis syndrome: an uncommon complication of fludarabine therapy of chronic lymphocytic leukemia. J Clin Oncol. 1998;16(7):2313-2320. 
59. Rioufol C, Coiffier B. Acute tumor lysis syndrome during oral fludarabine treatment for CLL- a rare event that might be observed more frequently in the future. Onkologie. 2008;31(4):157-158.

60. Calvo-Villas JM, Urcuyo BM, Umpierrez AM, Sicilia F. Acute tumor lysis syndrome during oral fludarabine treatment for chronic lymphocytic leukemia. Role of treatment with Fasturtec. Onkologie. 2008;31(4):197-199.

61. Ramachandran A, Majumdar G. Acute tumor lysis syndrome after oral fludarabine in a patient with chronic lymphocytic leukemia. Hematol J. 2004;5(6):528-529.

62. Dwyre DM, Holland PV. Transfusion-associated graft-versus-host disease. Vox Sang. 2008;95(2):85-93.

63. Kroger AT, Atkinson WL, Marcuse EK, Pickering LK; Advisory Committee on Immunization Practices (ACIP) Centers for Disease Control and Prevention (CDC). General recommendations on immunization: recommendations of the advisory committee on immunization practices (ACIP). MMWR Recomm Rep. 2006;55(RR-15):1-48.
64. Abzug MJ. Vaccination in the immunocompromised child: a probe of immune reconstitution. Pediatr Infect Dis J. 2009;28(3):233-236.

65. Bayer Canada Inc. Fludara ${ }^{\circledR}$, product monograph. Toronto, Canada: Bayer Canada Inc.; Oct 2, 2008.

66. Antisoma Research Ltd. Oral fludarabine, product monograph. London, UK: Antisoma Research Ltd; Dec 2008.

67. Lichtman SM, Etcubanas E, Budman DR, et al. The pharmacokinetics and pharmacodynamics of fludarabine phosphate in patients with renal impairment: a prospective dose adjustment study. Cancer Invest. 2002;20(7-8):904-913.

68. Lonardi S, Bortolami A, Stefani M, Monfardini S. Oral anticancer drugs in the elderly. Drugs Aging. 2007;24(5):395-410.

69. Ruddy K, Mayer E, Partridge A. Patient adherence and persistence with oral anticancer treatment. CA Cancer J Clin. 2009;59(1):56-66.

\section{Publish your work in this journal}

Drug Design, Development and Therapy is an international, peerreviewed open-access journal that spans the spectrum of drug design and development through to clinical applications. Clinical outcomes, patient safety, and programs for the development and effective, safe, and sustained use of medicines are a feature of the journal, which has also been accepted for indexing on PubMed Central. The manuscript management system is completely online and includes a very quick and fair peer-review system, which is all easy to use. Visit http://www.dovepress.com/testimonials.php to read real quotes from published authors.

Submit your manuscript here: http://www.dovepress.com/drug-design-development-and-therapy-journal 